Hor i zont al, verti cal, and radi al hi gh- energy parti cle di stributi on measur ement system in Lar ge Hel i cal Devi ce

\begin{tabular}{|l|l|}
\hline $\begin{array}{l}\text { jour nal or } \\
\text { publ i cat i on } \mathrm{titl} \text { e }\end{array}$ & Revi ew of Sci ent i f i c I nst r ument s \\
\hline vol une & Vol . 77 \\
\hline number & I ssue 10 \\
\hline page range & pp. 10E917- 1 10E917- 4 \\
\hline year & 2006-10-01 \\
\hline URL & ht t p: //hdl . handl e. net /10655/3785 \\
\hline
\end{tabular}




\title{
Horizontal, vertical, and radial high-energy particle distribution measurement system in Large Helical Device
}

\author{
T. Ozaki, P. Goncharov, S. Sudo, T. Seki, and LHD Experimental Group \\ National Institute for Fusion Science, Toki, Gifu 509-5292, Japan \\ S. Murakami \\ Department of Engineering, Kyoto University, Kyoto 606-8501, Japan \\ E. Veschev \\ The Graduate University for Advanced Studies, Hayama, Kanagawa 240-0193, Japan \\ J. Lyon \\ Oak Ridge National Laboratory, Oak Ridge, Tennessee 37831
}

(Received 2 May 2006; presented on 9 May 2006; accepted 26 June 2006; published online 6 October 2006)

\begin{abstract}
A horizontal, vertical, and radial high-energy particle distribution measurement system, which consists of the time-of-flight neutral particle analyzer (TOF-NPA), the silicon detector neutral particle analyzer (SD-NPA), and pellet charge exchange (PCX) analyzers, is successfully installed in the Large Helical Device (LHD). In a horizontal scan, it is interesting to measure the pitch angle distribution and to investigate the loss cone feature obtained by it. The pitch angle distribution from $40^{\circ}$ to $100^{\circ}$ can be obtained by horizontal scanning of the TOF-NPA during a long discharge of over $100 \mathrm{~s}$ sustained by the NBI2 (coinjection) at the magnetic axis of $3.6 \mathrm{~m}$. With a vertical scan, the heating deposition profile of the ion cyclotron resonance heating (ICH) has been discussed. The deposition profile can be obtained by the vertical scan of the SD-NPA. The region where the high-energy particle is generated agrees with the location of the resonance layer of the ICH. Similar results can be obtained by the PCX measurement, which gives radial information of the high-energy particle distribution in a short discharge. () 2006 American Institute of Physics. [DOI: 10.1063/1.2229270]
\end{abstract}

\section{INTRODUCTION}

It is important to study the generation mechanism of the high-energy particles in ion cyclotron resonance heating (ICH) plasmas in order to understand particle confinement. The resonance layer of the ICH is determined by a combination of the magnetic field and the frequency of the $\mathrm{ICH}^{1}$ Many highly energetic particles can be generated around the resonance layer. The theoretical resonance layer can be experimentally confirmed by a vertical scan of the neutral particle analyzer. ${ }^{2}$ However, it is difficult to find the resonance layer by scanning because the passive measurement is an integration of the sight line. The radial position of the resonance layer can be found by pellet charge exchange. ${ }^{3}$ By the combination of those diagnostics, an accurate measurement can be expected.

With the addition of the horizontal scanning of the analyzer, a three-dimensional measurement of the energetic particle is available. On helical devices, orbits of the generated particles in plasma are very complicated due to the magnetic field ripple. When these orbits are drawn in velocity space, some particles with large pitch angles are lost (loss cone). ${ }^{4,5}$ In LHD, three neutral beam injections (NBIs) are injected tangentially. However, if the slowing down of the incident particle by electron collision occurs, the particle with a large pitch angle actually will be generated due to the scattering between the particle and a plasma ion at several times of the plasma temperature. Therefore parts of them are not confined due to the loss cone. Similar phenomena also occur by direct perpendicular injection. To investigate the features of the loss cone, the pitch angle distribution of the high-energy particle, which is obtained by the horizontal scanning of the neutral particle analyzer, is very helpful.

To achieve the above purposes, we install the sixchannel silicon surface barrier detector with a vertical scanning system, the pellet charge exchange system, and the time-of-flight neutral particle analyzer with a horizontal scaning system in LHD.

\section{DIAGNOSTICS AND EXPERIMENTAL ARRANGEMENT}

A silicon surface barrier diode type neutral particle analyzer ${ }^{6}$ (SD-NPA) with a six-channel detector array can observe six different pitch angles from $35^{\circ}$ to $90^{\circ}$, as shown in Fig. 1. There are liquid nitrogen cooled detectors to avoid electrical noise with different sight lines. The minimum observable energy is $25 \mathrm{keV}$, which is determined by the thickness of the aluminum coating for light protection and the inactive layer of the silicon detector. The simultaneous six energy spectra with an energy resolution of $1.8 \mathrm{keV}$ can be obtained by traditional pulse height analysis. The electronics consist of the preamplifier cards in vacuum, the preamplifier boxes, the shaping amplifiers the ADCs, and the histogram 


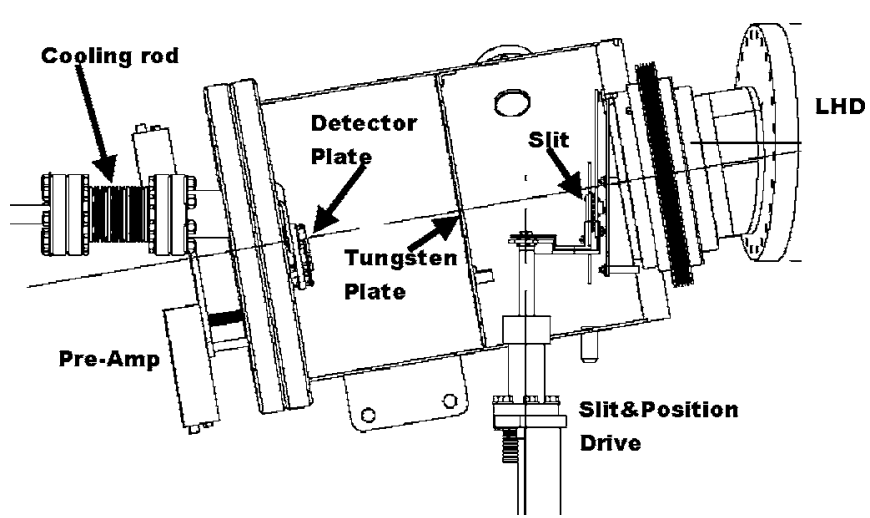

FIG. 1. Silicon surface barrier diode type neutral particle analyzer (SDNPA). The detector plate including inner preamplifier is connected with the liquid nitrogen cooled rod. A tungsten plate protects the detector from the $\mathrm{x}$-ray noise.

memories, which are controlled by a CAMAC system. The time resolution is normally $5 \mathrm{~ms}$ but $100 \mathrm{~ms}$ in the long discharges. Energy calibration has been carried out by using the Fe55 radio isotope $(5.9 \mathrm{keV})$. From this campaign a continuous vertical scan of $0.03^{\circ} / \mathrm{s}$ (scanning resolution $1^{\circ}$ ) can be made using an ultrasonic motor controlled by RS-232C. Therefore a two-dimensional measurement of the highenergy particle distribution has been possible during a long discharge, which is the advantage of LHD.

The radial profile of the energetic particles is observed by the pellet charge exchange (PCX) neutral particle measurement. The impurity pellet (polystyrene) is injected to the LHD plasma as mentioned in Ref. 7. The charge exchange neutral particle, which is created by charge exchange between the pellet ablation cloud and the high-energy ions localized around the pellet cloud, is observed from the backward of the pellet trajectory. Therefore we can translate the time trace of the energy spectrum into a radial profile by accurately monitoring pellet velocity. Particle detection has been done using a compact neutral particle analyzer with a carbon stripping film and a permanent magnet for energy analysis. This analyzer is calibrated by using the hydrogen atom source from 0.8 to $160 \mathrm{keV}$ in the Ioeffe Institute. The energetic proton is detected by the rectangular channeltron with an energy range of $0.8-168 \mathrm{keV}$ and with an energy resolution of several percent. The pellet velocity of $400-500 \mathrm{~m} / \mathrm{s}$ is monitored every shot by using two photodiodes. A spatial resolution of $4-5 \mathrm{~cm}$ can be expected with a time interval of $0.1 \mathrm{~ms}$.

The time-of-flight (TOF)-type neutral particle analyzer has been used for a horizontal scanning measurement of the energetic particle adding the SD-NPA. Its details including calibration and experimental configuration are described in Ref. 8. It has an energy range from 0.8 to $370 \mathrm{keV}$ (typical energy resolution of 7\%) with mass resolution using the time of flight. The scanning is performed with a pivot in front of the $10-\mathrm{O}$ port by using a motor, which is remotely controlled by RS232C. A charge-coupled device (CCD) camera monitors the scanning angle. The possible scanning angle is equivalent to the pitch angles from $40^{\circ}$ to $100^{\circ}$. Therefore a very accurate pitch angle distribution can be expected.

Figure 2 shows the experimental apparatus including the

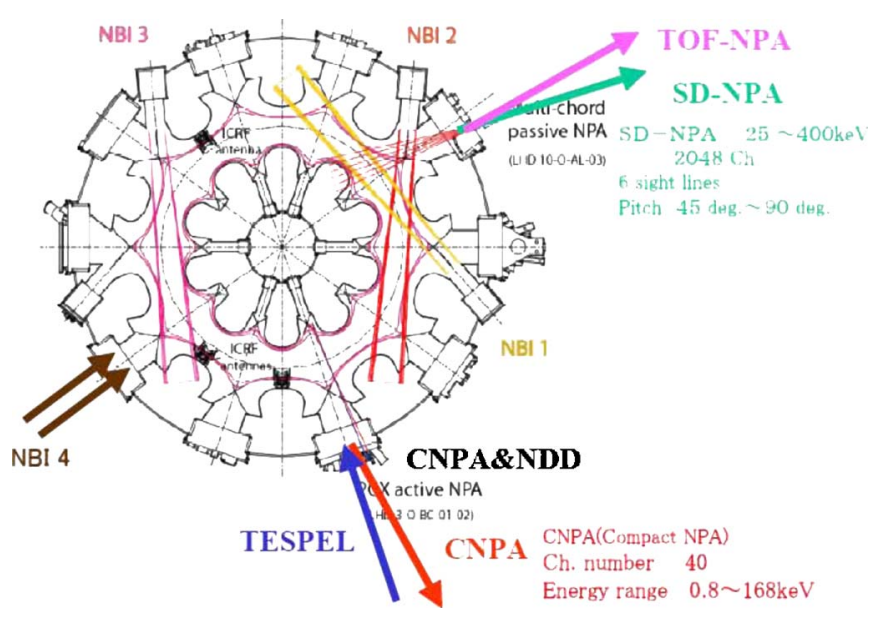

FIG. 2. The experimental apparatus. Three ICH antennas are installed at lower ports. NBI 1-3 and 4 are tangential injection and perpendicular injection, respectively. The SD-NPA and TOF-NPA are installed at the cross point of two NBIs as much flux can be expected.

relevant diagnostics and the plasma heating system. NBI 1-3 and 4 are tangentially injected and perpendicularly injected, respectively. SD-NPA and TOF-NPA are installed at the cross point of the two NBIs at that point since much flux can be expected.

\section{EXPERIMENTAL RESULTS OF VERTICAL SCAN AND PCX}

In LHD, a long discharge of over 50 min with a total input energy of $1.6 \mathrm{GJ}$ can be made by ICH. SD-NPA has an ability of the vertical scan of $0.03^{\circ} / \mathrm{s}$ by sliding the pinhole in order to obtain the vertical distribution of the neutral particle energy spectra. The deposition profile of ICH can be determined by the distribution. The resonance layer of the ICH is determined by the combination of the frequency and the magnetic field. In this discharge, the standard configuration of the resonance layer is chosen. Much high-energy flux can be observed near the resonance layer of the $\mathrm{ICH}$, as shown in Fig. 3. Figure 3 also shows the cross section of the resonance layer determined by calculation. Much flux is observed at the intersection between the condensed region of

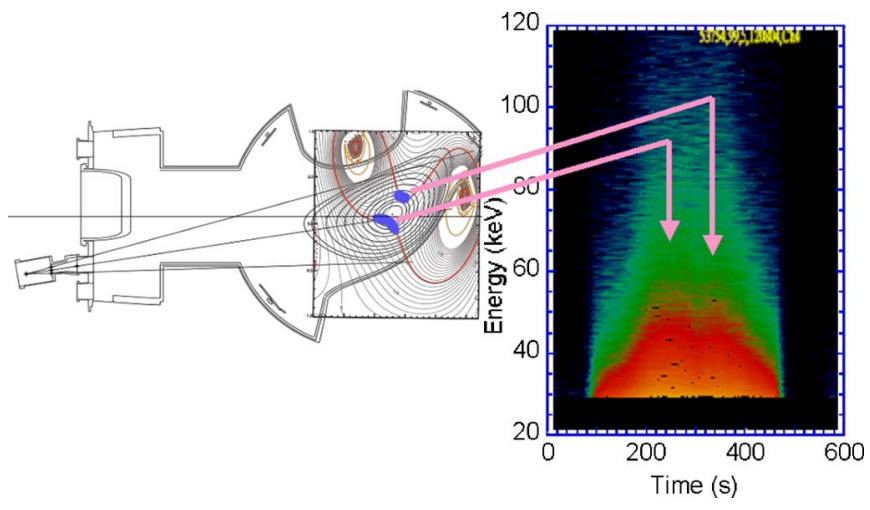

FIG. 3. The vertical scan of the SD-NPA. The SD-NPA is scanned from the bottom to top. During scanning, two peaks appear when the sight line crosses the strong resonance positions of the ICH. 


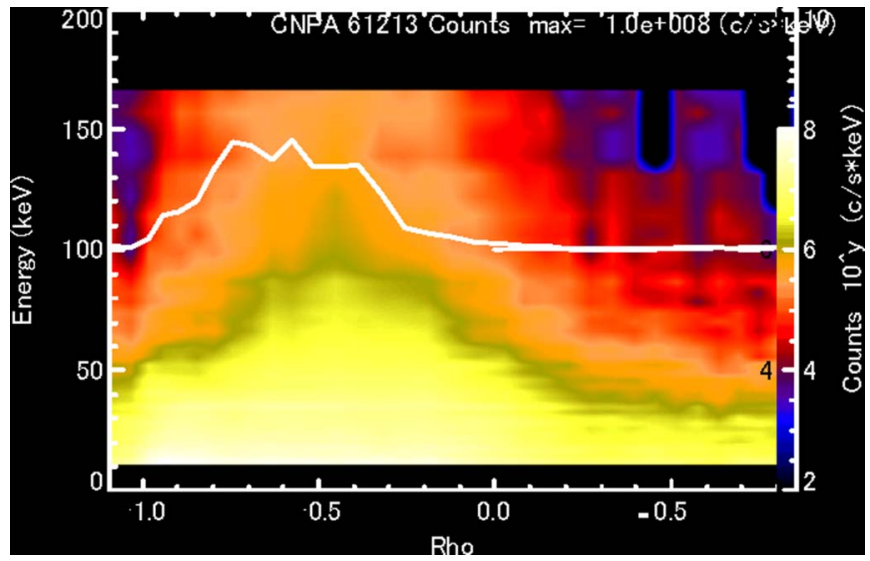

FIG. 4. The radial profile of the high-energy particle obtained from PCX. The color indicates the flux of the particle. The table of the flux is shown at the right side. The maximum flux can be observed at $\rho=0.5$ because that is the location of the resonance layer.

the resonance layer and the high plasma temperature region. The flux is larger at the perpendicular sight line rather than at the tangential sight line.

By the pellet charge exchange measurement, the radial position of the resonance layer is confirmed. The polystyrene pellet is injected in the ICH plasma. The penetration depth of the pellet is monitored to be $\rho=-0.2$ by the photomultiplier. Figure 4 shows the radial profile of the high-energy particle spectrum obtained from the time resolved energy spectrum. In this discharge, the resonance configuration is the same as that in the SD-NPA experiment. The flux maximum can be observed on the resonance layer at $\rho=0.5$ same as at SDNPA result in Fig. 3.

\section{HORIZONTAL SCAN}

To verify the loss cone feature in LHD, we study the pitch angle distribution of the trapped particles, which are produced by the deceleration of the NBI particle. The pitch angle distribution should be uniform when the energy of the NBI 1 is lost by collisions with plasma electrons and becomes an energy several times of the electron temperature. The particle with a large pitch angle has a drift motion and poloidal rotation. LHD has the capability of a long discharge and NBI 2 can support the plasma during a maximum of 3 min. The pitch angle distribution has been performed by the horizontally scannable TOF-NPA with the scanning speed of $0.7^{\circ} / \mathrm{s}$ in a steady state plasma.

Figure 5 shows the contour plot of the pitch angle distribution measured by the scanning of the TOF-NPA during a NBI long pulse discharge. Horizontal and vertical axes indicate the particle energy of hydrogen and the pitch angle, respectively. The color (or density) shows the flux of the particle. We must take into account the line integration of the particle flux in the experiment. However, trapped particles around a pitch angle of $90^{\circ}$ which are created by the scattering process are clearly found in Fig. 5. This means that there is no significant loss cone in pitch angle.

In long discharge experiments, it is very difficult to choose a different magnetic axis position because long discharges cannot be maintained except at $R_{\mathrm{ax}}=3.6 \mathrm{~m}$. We use

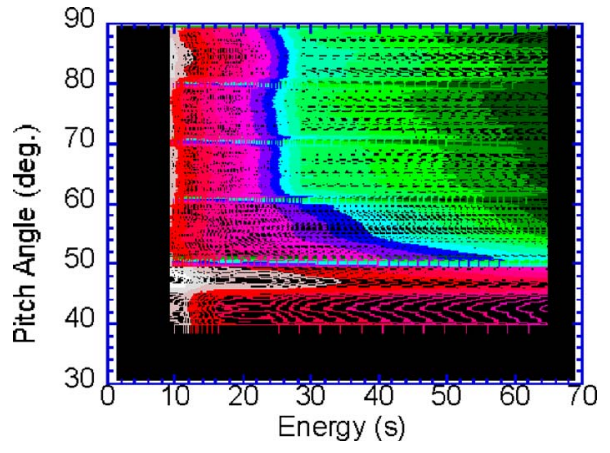

FIG. 5. The contour plot of the pitch angle distribution. The color indicates the flux of the particle. Trapped particles around the pitch angle of $90^{\circ}$ are clearly observed. The effect of the loss cone is not so large because the particle loss near $20 \mathrm{keV}$ is not significant.

the SD-NPA, which has six different sight lines in order to obtain the pitch angle distribution in a single short discharge. The pitch angle distribution of different magnetic axes can be obtained by using the SD-NPA. Figure 6 shows the contour plots of the pitch angle distribution at two different magnetic axes of $R_{\mathrm{ax}}=3.6$ and $3.75 \mathrm{~m}$. Horizontal and vertical axes indicate the particle energy of hydrogen and the pitch angle, respectively. The color (or density) shows the flux of the particle. By an inward shift of the magnetic axis, the number of trapped particles near the pitch angle of $90^{\circ}$ increases. This means that the trapped particles are well confined because the orbits of the trapped particles are more coincident with the magnetic surfaces when the magnetic axis moves inward.

\section{SUMMARY}

We show some examples of results by horizontal, vertical, and radial scanning of NPAs which are newly installed. The resonance layer of an ICH plasma can be observed using a vertical scanning technique with the SD-NPA The resonance layer in the radial direction is confirmed using a PCX technique. By using horizontal scanning of the TOF-NPA and the SD-NPA, the pitch angle distribution at $R_{\mathrm{ax}}=3.6 \mathrm{~m}$ and the magnetic axis dependence of the trapped particle confinement can be obtained. Through these diagnostics, a three-dimensional measurement system can be established in LHD.

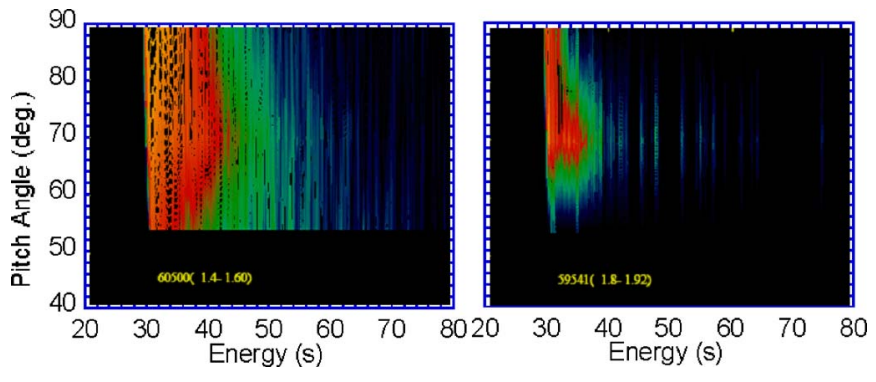

FIG. 6. The contour plots and spectra of the pitch angle distribution at two different magnetic axes. The perpendicular NBI 4 is injected to the LHD plasma. By inward shift of the magnetic axis, the number of trapped particles with a pitch angle near $90^{\circ}$ increases. 


\section{ACKNOWLEDGMENT}

This work was performed under NIFS05ULBB509, Grant Aid Nos. 17540475 and 18035013.

${ }^{1}$ T. Seki, R. Kumazawa, T. Mutoh, A. Fukuyama, K. Saito, Y. Torii, N. Takechi, and LHD Experimental Group, 30th EPS Conference on Controlled Fusion and Plasma Physics, 2003, p. 128.

${ }^{2}$ M. Wakatani, Stellarator and Heliotron Devices (Oxford University Press, Oxford, 1998), p. 262.
${ }^{3}$ H. Sanuki, J. Todoraki, and T. Kamimura, Phys. Fluids B, Plasma Phys. 2, 2155 (1990)

${ }^{4}$ V. V. Afrosimov, E. L. Berezovskii, I. P. Gladkovskii, A. I. Kislyokov, M. P. Petrov, and V. A. Sadovnikov, Sov. Phys. Tech. Phys. 20, 33 (1975).

${ }^{5}$ R. K. Fisher et al., Phys. Rev. Lett. 75, 846 (1995).

${ }^{6}$ J. Lyon, P. Goncharov, S. Murakami, T. Ozaki, D. E. Greenwood, D. A. Spong, and S. Sudo, Rev. Sci. Instrum. 74, 1873 (2003).

${ }^{7} \mathrm{P}$. Goncharov et al., Proceedings of the Ninth IAEA-TM, Takayama, Japan, p. 18

${ }^{8}$ T. Ozaki et al., Rev. Sci. Instrum. 71, 2698 (2000). 